\title{
PREGUNTAS COGNITIVAS Y METACOGNITIVAS \\ EN EL PROCESO DE APRENDIZAJE
}

\author{
Influencia de preguntas cognitivas y metacognitivas \\ en comprensión conceptual y en habilidad para resolver \\ problemas en ciencias
}

Ignacio Abdón Montenegro Aldana*

\begin{abstract}
The education is the process of the cultural promotion. The science is the foundation of the education. It's possibility to make education investigation with science focus. The center of the education process in the learning; which is the acquisition of knowledge and the evolution of this. The cognition is processing of mental representatives; and metacognition is the control of the cognition process. Inside this theorie frame, it have found the question like the activate and directive of the learn. The cognitive questions and metacognitive questions influence in the conceptual understanding and the ability for solver problems in science.
\end{abstract}

\section{RESUMEN}

Desde un enfoque científico de la investigación educativa, la educación se asume como un proceso de promoción cultural, en el cual la misma ciencia se constituye en su principal fundamento. La educación centrada en el aprendizaje implica concebir este proceso como adquisición de conocimiento y evolución del mismo. El conocimiento como representación de la realidad, requiere de la cognición como procesamiento de las representaciones mentales, y de la metacognición como el conocimiento de los procesos cognitivos. Dentro de este marco teórico se ha encontrado la pregunta como activadora y orientadora del aprendizaje: se ha podido establecer la influencia de preguntas cognitivas y metacognitivas en la comprensión conceptual y en la habilidad para resolver problemas en ciencias.

Palabras claves: Preguntas cognitivas, preguntas metacognitivas, comprensión conceptual, habilidad para resolver problemas.

\footnotetext{
* Docente investigador. Estudiante del programa de doctorado en Educación. Area en Ciencias Universidad Pedagógica Nacional.
} 


\section{INVESTIGACIÓN EDUCATIVA CON ENFOQUE CIENTÍFICO Y TECNOLÓGICO}

La educación se puede interpretar como un conjunto de hechos que ocurren al interior de las sociedades. Estos hechos pueden ser estudiados a la luz de formulaciones teóricas que permitan la elaboración de nuevos modelos de interpretación y modelos de intervención que orienten los procesos educativos a los deseos propios de las sociedades. En esto consiste el enfoque científico de la investigación educativa: en una contratación sistemática entre hechos y teorías, a fin de interpretar los acontecimientos y cualificar las concepciones.

El hombre como entidad inteligente no solo interpreta el mundo, también actúa sobre él para derivar su existencia. La historia del saber hacer es la historia de la tecnología. Siendo la educación en esencia actividad, requiere la incorporación cuidadosa de tecnología y del desarrollo sistemático de medios que permitan cualificar los hechos.

\section{La educación como proceso de promoción cultural}

Se habla de manera recurrente de la educación como formación integral del ser humano; del desarrollo de diferentes dimensiones que lo constituyen. Esta visión, válida socialmente, es a su vez limitada por su relativo estatismo, porque carece del dinamismo propio del hombre y de las diferentes sociedades que conforma.

En un intento por configurar una visión dinámica, la educación se puede concebir como un proceso de promoción cultural. Proceso, porque se realiza a través de etapas, de estados de transición sucesivos; promoción porque avanza de estados inferiores a estados superiores de desarrollo; y cultural porque se orienta a nuevos modos de actuación colectiva. En síntesis, el proceso de promoción cultural implica la racionalidad en su concepción moderna: pensar y actuar de la mejor manera posible, tanto en el plano individual como colectivo.

\section{La ciencia como fundamento principal de la educación}

La historia evolutiva del hombre ha sido su proceso de adaptación a la naturaleza y a la sociedad. En ese proceso ha generado conocimiento entendido como saber entender y saber hacer. Dentro del saber entender ha surgido la ciencia la cual tiene el doble carácter de proceso y resultado. La ciencia como proceso se ha caracterizado como un conjunto de actividades tendientes a la producción de conocimiento científico; es decir, aquel que busca la explicación de los fenómenos por relaciones causales entre los mismos. La ciencia como resultado es el conjunto de teorías explicativas del mundo a partir de leyes o principios inmersos en el mismo universo estudiado. El conocimiento científico es representación de la realidad con el más alto grado de validez. La ciencia se basa en la explicación y parte del supuesto de que el mundo es cognoscible; sus preguntas se caracterizan porque indagan cómo es la realidad. Formular una pregunta científica es preguntarle a la naturaleza sobre alguno de sus fenómenos (Schelessinger, 1999).

Por abordar el estudio sistemático de la realidad y considerar el conocimiento con grados relativos de validez, no como verdades absolutas, la ciencia se puede constituir en el fundamento principal de la educación del hombre; por ser él, objeto de conocimiento y a su vez, el sujeto que conoce. 


\section{El aprendizaje como adquisición y evolución del conocimiento}

Los estudios sobre los procesos educativos y la práctica de los mismos, se centran cada vez más en el aprendizaje. Qué aprendemos, cómo aprendemos y para qué; son cuestiones de permanente análisis, cuyas respuestas pueden contribuir de manera decisiva al mejoramiento de la calidad educativa. La consideración central es que la cognición y la metacognición constituyen dimensiones del aprendizaje.

El aprendizaje se puede concebir como el proceso a través del cual se adquiere conocimiento y éste evoluciona. La Sicología Experimental planteó la idea del aprendizaje como un cambio en el comportamiento; y en efecto, quien aprende algo nuevo, puede, en efecto, comportarse de manera diferente. En tal sentido, el aprendizaje lleva asociada la probabilidad de que un agente inteligente se comporte de una forma determinada, en condiciones determinadas. Por tal razón, el aprendizaje, no siempre implica cambio de conducta; y por ello, para la Ciencia Cognitiva, es ante todo un cambio en las estructuras conceptuales: conocimiento en evolución. Como el conocimiento declarativo es representación de la realidad tal representación se hace en algún tipo de estructura. La Neurociencia ha demostrado que todo aprendizaje genera cambios en la corteza cerebral en donde se forman nuevas sinapsis, algunas se fortalecen u otras se debilitan.

De acuerdo con Kandel y Jessel, "la mayoría de los aspectos de la conducta humana implican cierta forma de aprendizaje". De otra manera, muchos resultados de la conducta humana, son el resultado de la capacidad para aprender de la experiencia. En realidad, somos verdaderamente quienes somos, por lo que aprendemos y recordamos (Kandel, 1997). Estos investigadores clasifican el aprendizaje en dos formas: implícito y explícito. "Las formas implícitas son encubiertas y a menudo reflejas, no necesitan atención consciente, mientras que las formas explicitas requieren un conocimiento consciente". (kandel, 1997). Buena parte de los aprendizajes que se adquieren en la institución educativa y en especial los académicos, son explícitos. También allí ocurren aprendizajes implícitos, los que dan de manera directa por el simple hecho de interactuar con las personas y por estar en contacto con el ambiente institucional.

Para Robert Gagné el aprendizaje puede darse de manera intencional o incidental. En su artículo Dominios del Aprendizaje, lo presenta como el proceso en el cual se adquiere modificaciones en el conocimiento existente: habilidades, hábitos o tendencias a actuar (Gagné, 1989).

\section{El conocimiento como representación de la realidad y actuación en ella}

El conocimiento se puede abordar como la representación de la realidad y la capacidad para intervenir en ella. El cerebro es el medio de representación natural del conocimiento. (Leahey, 1998). Tales representaciones orientan buena parte de las acciones humanas. Para Helen Gagné existen dos tipos de conocimiento: declarativo y procedimental. Mediante el conocimiento declarativo se comprende el mundo, implica de manera preferente intervención de conciencia e intencionalidad. Buena parte de los conocimientos aprendidos en la institución educativa son de este tipo. El conocimiento procedimental es propiamente el saber actuar: en algunas ocasiones requiere el uso de la conciencia y en otros no. En este último caso se compone especialmente por actos reflejos. Sobre estas 
dimensiones del conocimiento, Maldonado ratifica el enfoque cuando retorna a Pirolli y Recker quienes "parten de la diferenciación entre conocimiento declarativo y procedimental; es decir, saber qué y saber cómo" (Maldonado, 2000). Señala que los sistemas de producción son un buen ejemplo para comprender el conocimiento procedimental.

\section{La cognición como procesamiento de representaciones mentales}

Teniendo en cuenta que el conocimiento es representación y que éste evoluciona a través del aprendizaje; en los humanos, "la mente es un tipo especial de computador y el proceso cognitivo es manipulación interna de representaciones". (van Gelder, 1996). En tal sentido, se puede considerar como un sistema dinámico porque genera transformaciones en dichas representaciones.

La cognición se puede llevar a cabo mediante estrategias. Una estrategia es un comportamiento constante que se mantiene invariable a través de un conjunto de actividades y que busca garantizar el logro de una meta u objetivo. Es una estructura decisión-acción. Se puede concebir entonces como el conjunto de reglas para asegurar la mejor decisión en cada momento a fin de lograr la cognición. Según Gagné las estrategias cognitivas son habilidades organizadas internamente que gobiernan el comportamiento individual en el aprendizaje. Se pueden tipificar estrategias cognitivas orientadas a la comprensión, la representación, la aplicación y la investigación. Mediante la comprensión, interpretamos hechos de la realidad y bases de conocimiento que se encuentran en agentes inteligentes naturales, artificiales o en dispositivos de memoria. Se efectúa a través de operaciones como la observación, la lectura, la escucha, la reflexión, el análisis, la comparación, la síntesis y la generalización. Mediante la representación medial, expresamos el conocimiento en cualquier forma bien sea verbal, textual, gráfica, icónica, diagrama o mapa conceptual. La representación implica comprensión y al representar algo se comprende mejor. La principal operación de estas estrategias es la modelación; es decir, la creación de un modelo para representar un sistema, un determinado concepto o cuerpo de conocimientos. Mediante la aplicación, utilizamos los conceptos para resolver ejercicios bien sean hipotéticos o reales. La ejercitación permite reforzar dichos conceptos y comprender que éstos se hallan relacionados con la realidad objetiva. La aplicación también puede ser en la solución de problemas cotidianos que hacen parte del contexto en el cual interactúa el estudiante. Mediante la investigación descubrimos nuevos problemas y los caminos para encontrar soluciones generando nuevo conocimiento. En la investigación se integra la comprensión, la representación y la aplicación.

Los conceptos son elaboraciones mentales, producto de la cognición, son categorías que expresan regularidades a través de un conjunto de atributos. Buena parte del conocimiento científico se expresa en forma de conceptos científicos. Comprender conceptos es identificar sus atributos y las regularidades que expresan, es encontrar las relaciones con otros conceptos.

La solución de problemas constituye otro de los campos en los cuales interviene la cognición. Un problema se asume como una meta con su correspondiente conjunto de pasos para alcanzarla. En el proceso de solución de un problema, la parte crucial, la constituye la comprensión del mismo; es decir, la identificación de los diferentes elementos que conforman su espacio y la manera como están relacionados. Luego viene la aplicación de una estrategia o identificación del camino para hallar la solución, la cual se alcanza cuando se ha logrado la meta deseada. 


\section{La metacognición como conocimiento del proceso cognitivo}

Una propiedad del ser humano es su capacidad para reflexionar sobre su propio conocimiento, para tener conciencia y control sobre su proceso cognitivo. Maldonado (Maldonado, 2000) afirma que la metacognición es el área de la ciencia cognitiva orientada al estudio de la regulación del propio aprendizaje. Su objeto de estudio es el conocimiento que el ser humano desarrolla sobre la manera como aprende, percibe, recuerda, piensa o actúa. La metacognición desarrolla conocimiento sobre el propio conocimiento. Stenberg considera la metacognición como una dimensión de la inteligencia porque es una habilidad crucial para el éxito educativo (Stenberg, 1994). La metacognición es también, un conjunto de comportamientos que pueden ser aprendidos mediante actividades académicas.

En otras palabras, la metacognición es un proceso asociado a la cognición humana que busca su optimización. Nelson y Narens proponen un modelo de los procesos metacognitivos compuesto por los niveles meta y objeto que interactúan mediante dos flujos de información: control y monitoreo. "El nivel objeto está constituido por los sistemas externos al sujeto cognoscente y se manifiesta como representaciones o modelos de esos sistemas y sus interrelaciones. El metanivel es una representación o modelo del conocimiento que se tiene a nivel objeto". (Maldonado, 2000).

Entre las investigaciones recientes sobre metacognición, el equipo del CIDUP. TECNICE de la Universidad Pedagógica Nacional, dirigido por el Doctor Luis Facundo Maldonado Granados, indagó sobre el papel de los activadores de juicios de metamemoria y de la sugerencia de estrategias en el aprendizaje autónomo (Maldonado, 2000). Sobre el proceso de solución de los problemas de diseño por parte de los estudiantes se distinguieron tres etapas: exploración, consolidación 1 y consolidación 2. Se observó que en la etapa exploratoria los sujetos poco se preocupan por el tiempo, por lo tanto, una preocupación temprana por la eficiencia no parece natural. Además, los juicios de metamemoria influyen en una etapa más temprana en la regulación de los procesos de búsqueda que las sugerencias de estrategias, las cuales actúan en etapas más tardías y cuando los sujetos han sobrepasado la etapa de descubrimiento. La combinación de activadores con estrategias genera un efecto de interacción positivo, dando como resultado puntajes intermedios y altos en el grupo que las utiliza. Los activadores de juicios de metamemoria como las sugerencias de estrategias tienden a ser más efectivos después de alguna experiencia de base en la solución de problemas comprobando que los estudiantes novicios tienen menor nivel de metacognición. El papel de las dos variables se incrementa con el avance en la curva del aprendizaje, pero también con otras condiciones del entrenamiento.

\section{La inteligencia artificial contribuye a la superación del déficit tecnológico de la Pedagogía}

De acuerdo con Luhmann, la Pedagogía se ha venido desarrollando con un déficit tecnológico estructural (Maldonado, 1997). La incorporación de inteligencia artificial puede contribuir a la superación de este déficit, en el sentido de aportar técnicas, métodos y materiales para apoyar los procesos de aprendizaje. 
Los esfuerzos de la Inteligencia Artificial IA están encaminados tanto a la construcción de entidades inteligentes como a su comprensión. Sus fundamentos se ubican en los planos de la Filosofía, las Matemáticas, la Psicología, la Lingüística y la Ingeniería Computacional. Sus trabajos se realizan con cuatro enfoques: actuar como humano, pensar como humano, actuar racionalmente, pensar racionalmente (Russell, 1996).

En el campo educativo, los sistemas inteligentes tuvieron como sus antecesores a los micromundos, concebidos como mundos restringidos que se pueden explorar de manera indefinida; luego aparecieron los simuladores como dispositivos que representan la dinámica de un sistema que de otra manera resultaría muy costoso o riesgoso. "La simulación de un sistema es la operación de un modelo (simulador) el cual es una representación del sistema. Este modelo puede sujetar-se a manipulaciones que serían imposibles de realizar, demasiado costosas o imprácticas. La operación de un modelo puede estudiarse y con ello inferirse las propiedades concernientes al comportamiento del sistema o subsistema real". (Naylor, 1971). En un momento posterior, aparecen los sistemas expertos que representan el conocimiento del especialista. A partir de ellos, se desarrollan los tutores inteligentes como dispositivos que emulan el proceso de aprendizaje de un novato con la ayuda de un experto. Su estructura permite la modelación de cuatro componentes: el dominio de conocimiento, el estudiante, el pedagogo y la interfaz. Recientemente, los sistemas inteligentes han sido enriquecidos conceptualmente con la visión agente-ambiente: la existencia de agentes que perciben y actúa en ambientes (Wenger, 1987). La programación orientada a objetos es una nueva concepción acerca del proceso de solución de problemas de programación, es la simulación de un universo modelo (Budd, 1994). Considera el programa como una colección de agentes con niveles de autonomía, llamados objetos y por tal razón se constituye en una de las mejores alternativas para implementar sistemas inteligentes.

\section{El conocimiento se puede representar en lógica de primer orden}

En los sistemas inteligentes se habla de un agente basado en conocimiento como aquel capaz de actuar sobre el mundo con base en representaciones del mismo. Esto es posible si el agente posee una base de conocimientos y un motor de inferencia. Para que el agente pueda razonar y producir inferencias válidas es necesario expresarle el conocimiento en un lenguaje de representación computacional. Todo lenguaje de representación posee sintaxis y semántica; si éstas son precisas, el lenguaje es una lógica. Existen varios lenguajes de representación computacional de conocimiento, uno de ellos y de propósito general es la lógica de primer orden. Éste considera al mundo constituido por objetos con propiedades específicas y con relaciones entre sí. La forma simbólica de representar el conocimiento en este lenguaje es a partir de oraciones atómicas, formadas por signos de predicados que se aplican a una lista de términos (Russell, 1996).

\section{LA PREGUNTA COMO ACTIVADOR DEL APRENDIZAJE}

La pregunta es un recurso usado de manera cotidiana tanto por docentes como por sujetos que aprenden. ¿Cuál es la función de la pregunta en los procesos de aprendizaje? ¿Qué es una pregunta? Desde el plano de la lógica, una pregunta es un enunciado sin valor de verdad puesto que no se puede afirmar si es falso o verdadero. La pregunta indaga por algo incierto, pero al mismo tiempo presupone un nivel de conocimiento de Digitalizado por RED ACADEMICA 
aquello que se desconoce, de no ser así, ni siquiera podría formularse. Por esto, una manera de caracterizar la pregunta es el planteamiento certero de una incertidumbre; es el punto de partido para ingresar al mundo de lo desconocido, para encontrar la información que se requiere. Buscar información para poder actuar mejor, es una necesidad de las entidades inteligentes. La teoría científica de la información afirma que la información es reducción de incertidumbre. En el plano de la ciencia, la pregunta es la forma como se expresan los problemas. A nivel pedagógico, se constituye en un facilitador de los procesos comunicativos, pedagógicos y científicos: un activador del aprendizaje.

Indagando sobre la función pedagógica de la pregunta se realizaron dos trabajos previos: En el primero de ellos se examinaron los efectos de la pregunta sobre el proceso de aprendizaje en ciencias (Montenegro, 1997). Se comprobó que las preguntas contribuyen de manera sistemática a la apropiación conceptual en los estudiantes y favorece también su habilidad para formular preguntas. Se notó, además, un efecto de saturación y un incremento notable de la entropía: las preguntas facilitan la ganancia de información pero al mismo tiempo generan incertidumbre. En el segundo, se estudió la función de la Pregunta en los procesos de razonamiento en ciencias: de qué manera contribuye en los procesos de aprendizaje y, si es, un activador de estos procesos. [Montenegro, 1999]. En cuanto a los significados implícitos en la forma de preguntar se detectaron tres propósitos en la formulación de preguntas por parte del profesor: incentivar la participación de los estudiantes, valorar sus conceptos y controlar el ambiente de clase. Se pudo evidenciar, aunque de manera leve, las funciones cognitivas y metacognitivas de la pregunta: Se identificaron preguntas orientadas a la recordación, al análisis y a la comparación, que por estar orientadas al objeto de conocimiento se pueden denominar preguntas cognitivas. También se detectaron preguntas dirigidas a la valoración de los conceptos del estudiante y al control del ambiente de aprendizaje, por estar orientadas a la regulación de la cognición, se pueden denominar preguntas metacognitivas.

\section{Las preguntas cognitivas activan y dirigen el proceso de la cognición}

Teniendo en cuenta que mediante la cognición se procesan representaciones de la realidad, las preguntas pueden considerarse el vehículo a través del cual se activan y se orientan las estrategias cognitivas como la comprensión, la representación, la aplicación y la investigación. En tal sentido, se consideran preguntas cognitivas tipologías como las siguientes: ¿Cómo es? ¿Qué ocurrió? ¿Cómo ocurrió? ¿Por qué? ¿Cuál es la explicación? ¿En qué se parece? ¿En qué se diferencia? ¿Qué pasaría si? ¿Qué relación existe? ¿Cuál es la conclusión? ¿Cómo representar? ¿Qué factores intervienen? ¿Cómo demostrar? ¿Cómo resolver? ¿Qué aplicación se puede dar? ¿Qué inquietudes surgen? ¿Cómo superar la contradicción?, etc.

\section{Las preguntas metacognitivas facilitan la toma de conciencia del proceso cognitivo y contribuyen a regularlo}

De acuerdo con la formulación teórica sobre la metacognición, las preguntas de este tipo cumplen la función de activadores de juicios de metamemoria y se orientan al monitoreo, bien sea retrospectivo, recurrente o prospectivo. Se pueden definir tipologías como las siguientes: ¿Qué tan acertada fue su respuesta? ¿Eligió la ruta correcta? ¿Qué tan fácil fue resolverlo? ¿Cuántos intentos empleó? ¿Cumplió el objetivo? ¿Está empleando el tiempo previsto? ¿Se encuentra seguro de estar aprendiendo? ¿Se siente 
satisfecho con los logros obtenidos? De $\mathrm{N}$ preguntas, ¿cuántas responderá en forma correcta? ¿Cuánto tiempo empleará en resolver el problema?

\section{Las preguntas cognítivas y metacognitivas influyen sobre el aprendizaje}

Con el fin de indagar la influencia de preguntas cognitivas y metacognitivas sobre el aprendizaje en ciencias, se diseñó un prototipo de sistema el cual fue aplicado a un grupo de estudiantes, y los resultados fuero sometidos a un análisis factorial de varianza. (Montenegro, 2001).

\section{Diseño de un prototipo de software para implementar preguntas cognitivas $y$ metacognítivas}

El propósito del software es apoyar al estudiante en los procesos comprensión de conceptos y desarrollo de habilidades para resolver problemas, referidos ambos a la dinámica del oxígeno y del gas carbónico atmosféricos y su relación con algunos procesos naturales. La estructura general del programa es un sencillo prototipo que intenta aplicar el concepto de tutor inteligente clásico. Así pues, el modelo del dominio contiene la representación del conocimiento la cual se hace a través de un pequeño sistema experto y de un micromundo. El modelo pedagógico define las relaciones entre el dominio y el estudiante para que éste aprenda. El modelo de estudiante registra el nivel de logro dentro del proceso de aprendizaje. El modelo de la interfaz permite la comunicación entre el programa y el estudiante.

El micromundo es un dispositivo mediante el cual, a manera de juego, el usuario ejerce una sensación de control sobre la dinámica del oxígeno y del gas carbónico atmosférico, a través de su acción sobre agentes fotosintéticos y agentes productores de gas carbónico. Para ello se le plantea un problema de desequilibrio, el cual debe resolver de manera gráfica. El sistema experto representa de manera estructurada los conceptos que hacen parte del dominio; está conformado por la base de conocimientos y el motor de inferencia. La base de conocimientos está conformada por los conceptos principales y los subconceptos, expresados de tal manera que puedan ser entendidos por la computadora y que esta pueda razonar sobre ellos. Se sigue la lógica de primer orden, y en el programa se hallan codificados como listas o tablas consignadas en bases de datos. El motor de inferencia es el conjunto de reglas para plantear y resolver preguntas utilizando la base de conocimientos representada. El motor de inferencia permite que el sistema le formule preguntas al estudiante y que el estudiante le haga consultas al sistema.

El modelo del estudiante se define como el estado de conocimiento del estudiante de acuerdo con el dominio. Para ello, la variable a considerar es Dominio de Conocimiento la cual se establece mediante la evaluación de cada uno de los conceptos. El modelo pedagógico es la forma como el sistema orienta al estudiante para que logre cada uno de los objetivos. Es un modelo bastante elemental que opera como un sistema de decisiones que se determinan en función de la variable considerada en el modelo del estudiante.

El estudiante, primero conoce el objetivo, se le presenta un texto explicativo sobre el concepto, luego el sistema, utilizando el experto le hace preguntas cognitivas, se las valora, le ofrece refuerzo y dependiendo del puntaje obtenido continúa o se devuelve Digitalizado por RED ACADEMICA 
entregando un nuevo refuerzo correspondiente. Si el puntaje obtenido $\mathrm{P}$ es inferir a un valor $\mathrm{V}$ tomado como parámetro de dominio conceptual, entonces se considera que no ha cumplido el objetivo y por lo tanto el estudiante es llevado a que lea el texto nuevamente. Si $\mathrm{P}$ es igual o superior a $\mathrm{y}$, continúa respondiendo una pregunta metacognitiva que le formula el sistema. El modelo de la interfaz se presenta como un conjunto de procedimientos para garantizar la comunicación con el usuario de manera pertinente e interactiva.

Para asegurar que el sistema registre la influencia de preguntas cognitivas y metacognitivas en el proceso de aprendizaje, sus componentes se organizan de la siguiente manera: 1) pruebas de entrada: conceptual y habilidad para resolver problemas; 2) sesión de estudio de siete módulos con apoyo de preguntas cognitivas y metacognitivas; 3) pruebas de salida: conceptual y habilidad para resolver problemas.

\section{Diseño metodológico con análisis factorial de varianza}

El problema se estructuró de la siguiente manera: ¿Existe influencia de las preguntas cognitivas y metacognitivas en los procesos de comprensión conceptual y en la habilidad para resolver problemas? ¿Existe un efecto conjunto de los dos tipos de preguntas sobre cada una de las dimensiones del aprendizaje? ¿Existe correlación entre comprensión conceptual y habilidad para resolver problemas?

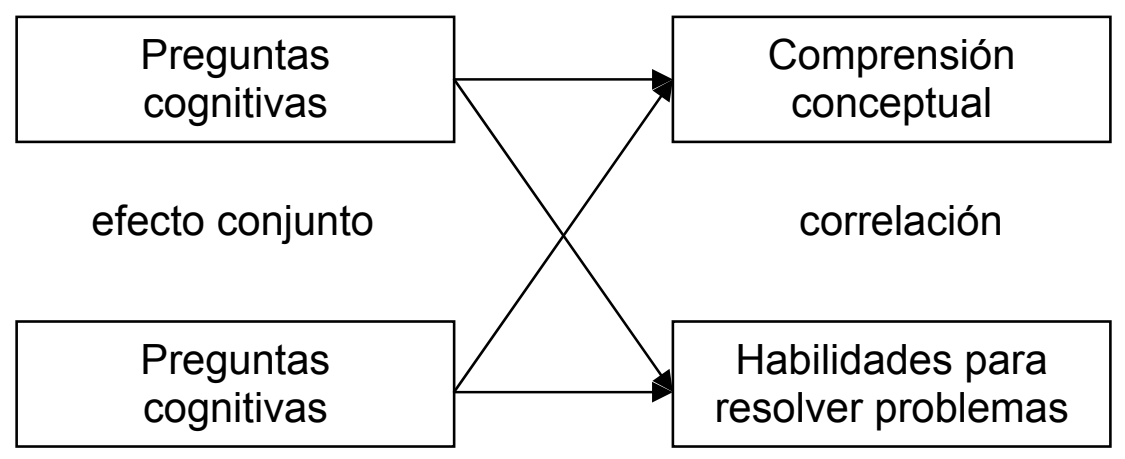

Para examinar de manera sistemática las variables anteriores, se organizan 4 grupos de estudiantes, conformados mediante una partición cruzada entre la utilización de preguntas cognitivas y metacognitivas.

El software se adecuó a cada uno de los grupos, en cuatro versiones diferentes. El tratamiento estadístico se realizó siguiendo la metodología de análisis factorial de varianza, uno de los métodos estadísticos del análisis multivariable cuyo propósito consiste en analizar medidas múltiples de $\mathrm{N}$ individuos. "En el análisis de varianza factorial dos o más variables independientes varían independientemente o interactúan entre sí para producir una variación en una variable dependiente. Es un método estadístico que analiza los efectos independientes e interactuantes de dos o más variables independientes sobre una variable dependiente.

Las preguntas cognitivas y metacognitivas influyen de manera distinta sobre el aprendizaje

Los estudiantes interactuaron tres veces con el software obteniéndose 6 mediciones de Comprensión Conceptual C, y 6 mediciones de Habilidad para Resolver Problemas H. 
De acuerdo con el análisis factorial de varianza se obtuvieron los siguientes resultados:

1. Los estudiantes que usan preguntas cognitivas $P C$ obtienen mejores rendimientos en comprensión conceptual $\mathrm{C}$, y en habilidad para resolver problemas $\mathrm{H}$ en ciencias, frente a estudiantes que no usan este tipo de preguntas.

2. Los estudiantes que usan preguntas metacognitivas PM tienden a obtener mejores rendimientos en comprensión conceptual $\mathrm{C}$ en ciencias, $\mathrm{y}$ en habilidad para resolver problemas $\mathrm{H}$, frente a estudiantes que no usan este tipo de preguntas; sin embargo, las diferencias no son significativas.

3. Existe una interacción baja entre preguntas cognitivas PC y metacognitivas PM sobre la comprensión conceptual $\mathrm{C}$ y sobre habilidad para resolver problemas $\mathrm{H}$ en ciencias, entre estudiantes que usan estos tipos de preguntas. Existe correlación, en un grado aún no establecido, entre comprensión conceptual $\mathrm{O}$ y habilidad para resolver problemas $\mathrm{H}$ en el proceso de aprendizaje en ciencias, en función de las dos variables anteriores.

El efecto positivo y significativo de las preguntas cognitivas sobre la comprensión conceptual se puede explicar de la siguiente manera: La comprensión conceptual se puede entender como la identificación del tipo de relaciones que se dan entre los elementos que componen un concepto y también de las relaciones de los conceptos entre sí. Las preguntas de orden cognitivo actúan como una especie de catalizador que activa el proceso de razonamiento a través del cual salen a la luz tales relaciones.

El efecto también positivo de las preguntas metacognitivas se explica en el poder que ejerce el conocimiento del proceso cognitivo dentro del aprendizaje. Las razones por las cuales no se obtuvo un efecto significativo guardan relación con la naturaleza de la metacognición: Ésta surge cuando el proceso de cognición ha llegado a cierto nivel de consolidación, lo cual significa que se requieren mayores niveles de comprensión conceptual para que el efecto de la metacognición se haga notorio. Probablemente en un período de aprendizaje más largo se tengan efectos significativos.

El efecto positivo de las preguntas cognitivas sobre la habilidad para resolver problemas obedece al efecto sobre la comprensión conceptual que a su vez permite entender el problema y elaborar estrategias de solución. Sin embargo, este efecto es limitado; ello se explica debido a la naturaleza del problema y a la forma cómo éste se plantea dentro del software.

El efecto de las preguntas metacognitivas sobre la habilidad para resolver problemas resultó positivo, aunque no significativo. Por ello, el efecto de interacción de las dos variables independientes fue bastante bajo.

\section{La interactividad puede ser el factor principal que determina el aprendizaje}

Con los anteriores resultados se re-afirma la pregunta como activador y orientador de los procesos de razonamiento. Esto conduce a pensar que la interactividad, en general, puede ser el factor principal que determina el éxito en el aprendizaje. 
La comprensión conceptual está más ligada al conocimiento declarativo y por ello la pregunta cognitiva ejerce mayor efecto, porque activa y orienta el procesamiento de información. En Ciencias, la habilidad para resolver problemas, en los cuales se conoce la meta y se dispone de unos medios para alcanzarla, depende de conocimiento declarativo y conocimiento procedimental. Por ello la comprensión conceptual y el entrenamiento son factores fundamentales para hallar soluciones eficientes. La metacognición es un proceso que requiere cierto nivel de consolidación conceptual para que pueda ejercer efectos favorables y significativos sobre el aprendizaje.

Con respecto al tiempo, el aprendizaje no es un proceso de incrementos; tampoco, es caótico. Es un proceso dinámico con ascensos y descensos, con una tendencia creciente a la adquisición de logros. Los factores que intervienen en este tipo de comportamiento son materia de investigación.

Como perspectivas, la separación teórica entre cognición y metacognición, constituye un buen recurso metodológico para comprender mejor la dinámica del proceso del aprendizaje. Se ha usado la pregunta como un elemento que orienta los procesos de razonamiento; también se ha empleado un micromundo en el cual se pueden plantear problemas; es decir, metas con sus correspondientes medios para lograrlas. Tanto la pregunta como el micromundo se constituyen en elementos que favorecen la interactividad del sujeto que aprende. Por ello, sería conveniente indagar sobre el efecto de otros factores como la observación, el análisis de hechos, la experimentación y la representación medial de conocimiento. El estudio sistemático de estos y otros factores, su grado de influencia, la relación entre ellos; su utilización con el apoyo de preguntas y en entornos computacionales agradables, abrirá la posibilidad para el diseño de modelos de aprendizaje de mayor efectividad.

\section{REFERENCIAS BIBLIOGRÁFICAS}

Budd, Timothy (1994). Programación Orientada a Objetos. Wilmington de la Ware: Addison Wesley Iberoamericana. Versión española de Judith García González. Título Original. An Introduction to Objected Promaming.

Gagné, Robert. M. (1989). 50 years of research. Domains of learning. Florida: Learning Systems Institute.

Kandel, Eric y otros. (1997). Neurociencia y Conducta. Madrid: Prentice Hall. Traducida del inglés por Pilar Herrera de Tejada y otros. Título original: Essentials of Neural Science and Behavior Apleton.\& Lange, 703, 716.

Kerlinger, Fred N. (1988). Investigación del Comportamiento. México: Mc Graw Hill Interamericana, 257.

Leahey, Thomas H.; Harris, Richard J. (1998). Aprendizaje y Cognición. Madrid: Prentice Hall International. Título original en inglés: Learning and Cognition, 145, 166, 289.

Maldonado G., Luis F. (1997). La pedagogía en el Tercer Milenio: Personalización e Informática. Bogotá: Publicación Universidad Pedagógica Nacional. 
Maldonado Granados Luis Facundo; otros. (2000). Metacognición y razonamiento espacial en juegos de computador. Bogotá: Publicación Universidad Pedagógica Nacional e Instituto para la investigación educativa y desarrollo pedagógico DEP. 1-32.

Montenegro A., Ignacio A. (1997). La Construcción de Conocimiento Científico como Proceso Creativo. Apropiación Conceptual en los Niños a través de una Estrategia Basada en Generación de Preguntas. Bogotá: Universidad Pedagógica Nacional. Tesis para la obtención del título en la maestría: Tecnologías de la Información Aplicadas a la Educación. En el Centro de Documentación de la Universidad, 132, 133.

Montenegro A., Ignacio A.. (1999). Función de la Pregunta en los Procesos de Razonamiento en Ciencias. Estudio Etnográfico. Bogotá: Inédito. Informe Universidad Pedagógica Nacional, 1.

Montenegro A.; Ignacio A. (2001). Influencia de preguntas cognitivas y metacognitivas en el proceso de aprendizaje en ciencias con el apoyo de un sistema inteligente. Bogotá: Inédito. Universidad Pedagógica Nacional. Proyecto de tesis para obtener el título de Doctorado en Educación. Area: Educación en ciencias. Línea: Inteligencia artificial y procesos de razonamiento en ciencias.

Naylor, Balirtfy y otros. (1971). Técnicas de Simulación en Computadores. Barcelona: Editorial Limusa.

Russell, Stuart; Norving, Peter. (1996) Inteligencia Artificial. Un En foque Moderno. Madrid: Prentice Hall. Traducido del inglés: Artificial Intelligence a modern approach, 3-17, 161-224.

Schlessinger B, Allen. (1999). Introdution to Scientific Explanation. México: Mc Graw Hill.

van Gelder, Timothy. "Dynamics and Cognition. (1996) En: Mind Disegn II. Edited by John Haugeland. A Bradfor Book. Massachusettes: The MIT Pres, 421.

Wenger, (1987. Artificial intelligence and Tutoring Systems: computational and cognitive approaches to communication of knowledge. Los Altos. C.A. Morgan Kaufmann Publishers, Inc. 